\title{
Visão do Paciente Quanto à Participação do Residente no Exame Proctológico em Ambulatório
}

\author{
Patient View About the Proctological Exam Assisted by the Resident in Outpatient Clinics
}

ISABELAPESSOAELIAS; ANTONIO LACERDAFILHO ${ }^{1}$; ELIANE SANDER MANSUR ${ }^{1}$; FREDERICO GUSMÃO

CÂMARA; KANTHYA ARREGUY DE SENA

${ }^{1}$ Membro Titular da S.B.C.P.

ELIAS IP; LACERDA FILHO A; MANSUR ES; CÂMARA FG; SENA KA. Visão do Paciente Quanto à Participação do Residente no Exame Proctológico em Ambulatório Rev bras Coloproct, 2006;26(4): 389-393.

RESUMO: Objetivos: $O$ acompanhamento ambulatorial de pacientes e a participação no exame proctológico são essenciais ao aprendizado do residente de coloproctologia. Entretanto pode haver relutância por parte dos pacientes em relação à presença do residente durante sua consulta e exame físico. Este estudo visa avaliar a visão do paciente quanto a este aspecto do aprendizado prático do residente de coloproctologia. Métodos: Cem pacientes consecutivos responderam , de forma voluntária e anônima a um questionário após a realização de exame proctológico com a aprticipação de residentes em coloproctologia em ambulatório de instituição privada. Foram investigados, sexo, idade, cor, estado civil e nível educacional e sócio-econômico, assim como a visão do paciente em relação à presença de residentes durante o exame proctológico, procurando-se identificar razões para a sua aceitação ou recusa durante a consulta. As respostas foram analisadas usando-se teste do qui-quadrado e o teste t de Student. Resultados: Observou-se que $87 \%$ dos pacientes aceitaram bem a participação do residente no exame proctológico, enquanto $1 \%$ respondeu que recusaria sua presença. Onze por cento se mostraram indiferentes em relação à presença do residente e um paciente não respondeu. Não houve diferença significativa em relação às variáveis estudadas no que diz respeito à presença do residente durante o exame proctológico, exceto para estado civil, uma vez que uma taxa maior de pacientes casados aceitou o residente. A principal razão para a aceitação foi contribuir para a formação médica, enquanto que a recusa esteve relacionado à perda da privacidade. Conclusão: De um modo geral, os residentes são bem aceitos no ambulatório de coloproctologia em uma instituição privada. O conhecimento das razões para sua aceitação ou recusa podem favorecer posturas que facilitem a aceitação e minimizem a recusa.

Descritores: residente, exame proctológico, educação médica.

\section{INTRODUÇÃO}

A educação médica apóia-se sobre dois palres: a aquisição do conhecimento teórico e das habilidades práticas. A residência médica, etapa fundamental para a formação do especialista, tem no conhecimento prático a base de seu aprendizado. Para tanto, o atendimento ambulatorial de pacientes e a participação no seu exame físico são essenciais para a adequada formação do médico residente. Entretanto, em especialidades como a coloproctologia, o exame físico é profundamente íntimo, lidando com área do corpo humano, na qual imperam preconceitos e tabus. Desta forma, pode haver relutância, nem sempre evidente, por parte dos pacientes à participação do residente no exame proctológico em regime ambulatorial. A literatura médica fornece poucas informações sobre este assunto, não se conhecendo os fatores que poderiam influenciar a aceitação ou recusa do paciente com relação à presença do residente durante o exame proctológico.

Este estudo tem como objetivo avaliar a visão do paciente neste aspecto, determinando os fatores que poderiam influenciar para a aceitação ou recusa da presença do residente durante a consulta e o exame proctológico.

\section{PACIENTES E MÉTODOS}

Cem pacientes consecutivos forma submetidos à consulta e exame proctológico em ambulatório, estando sempre presentes o preceptor e um residente. Ambos poderiam ser do mesmo sexo, ou do sexo masculino e

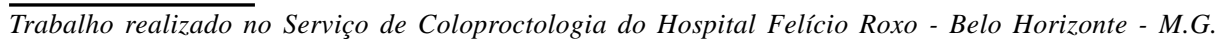

Recebido em 14/06/2006

Aceito para publicação em 20/07/2006 
feminino ou vice-versa. O paciente era informado quanto à presença do residente, sendo que a permissão para a participação do mesmo durante a consulta, não era rotineiramente solicitada. Caso fosse do desejo do paciente, o residente se retirava da sala de exame. A consulta e o exame proctológico eram realizados pelo preceptor com acompanhamento do residente ou praticados por este sob a supervisão direta do preceptor. O exame consistia em ectoscopia anal, toque retal, anuscopia e retossigmoidoscopia rigida e no exame da região abdominal. Após o término da consulta, o paciente era informado a respeito da presente investigação, sendo convidado a responder o questionário, de forma voluntária e anônima.

Deste questionário contavam perguntas informativas sobre sexo, idade, cor, estado civil, nível- sócio econômico, nível de instrução educacional e a visão do paciente quanto ao contexto de aprendizado e a participação do residente em sua consulta (Tabela 1). A caracterização da amostra é apresentada em percentuais simples. A comparação entre os grupos de respostas positivas ou negativas foi realizada através do teste de qui-quadrado ou de Fisher e para comparação de médias foi ultilizado o teste $t$ de Student.

Valores de $p<0,05$ forma considerados significativos.

\section{RESULTADOS}

Cem questionários foram preenchidos. O grupo estudado consistia de 54 mulheres e 46 homens com

Tabela 1 - Questionário de pesquisa da visão do paciente quanto a presença do residente na consulta e exame proctológico.

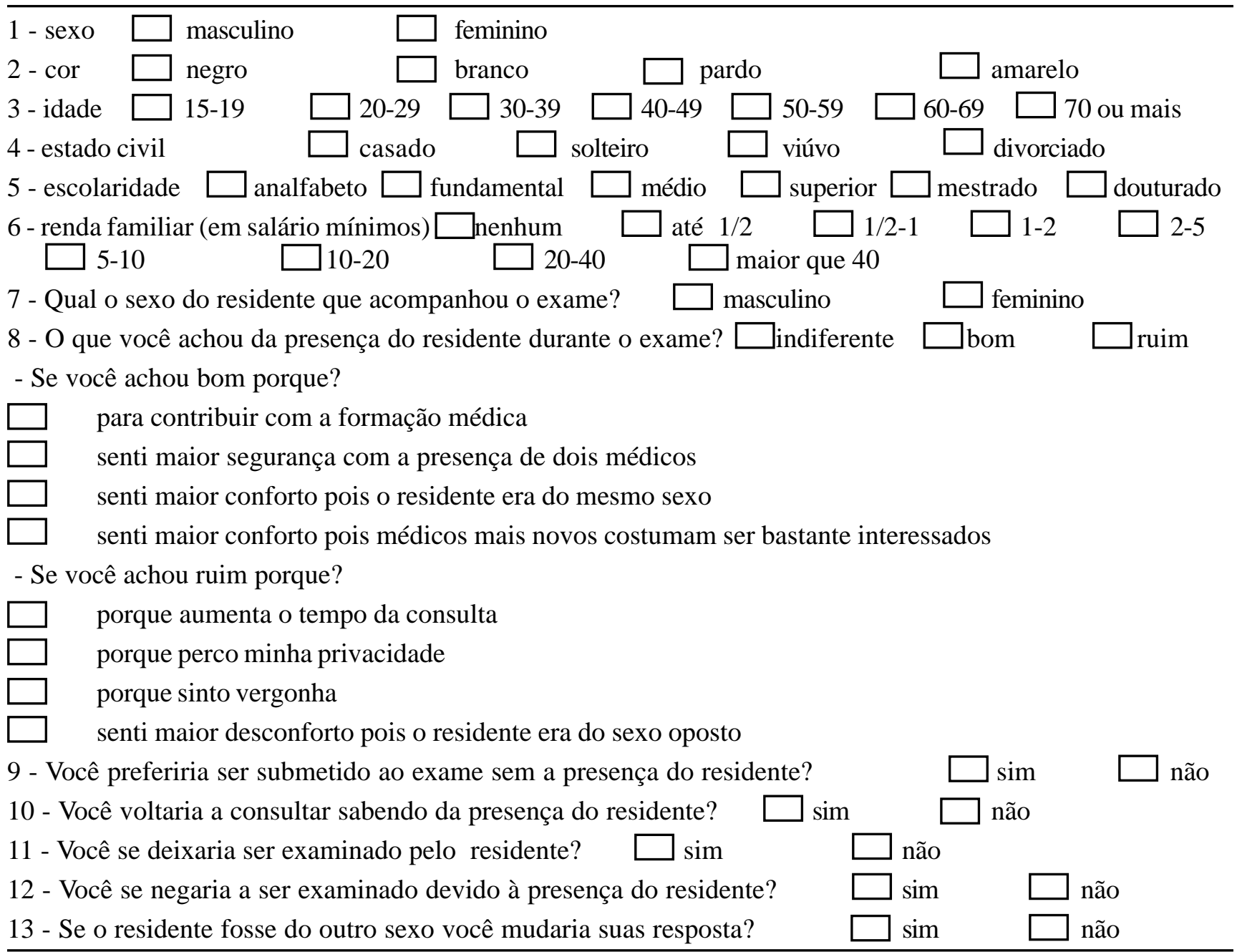



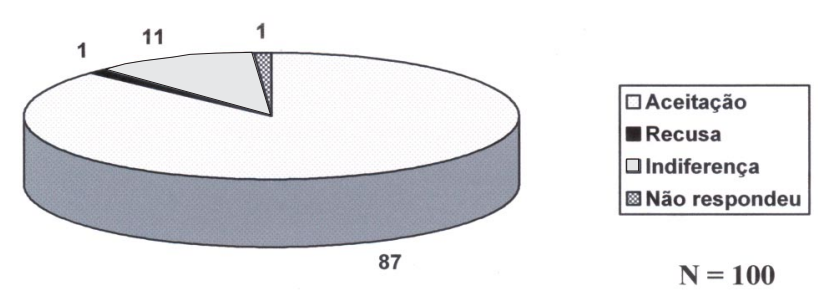

Figura 1 - Distribuição dos pacientes de acordo com a aceitação, recusa ou indiferença em relação à presença do residente durante o exame proctológico.

idade entre 30 e 49 anos, sendo a maioria de pacientes brancos $(67 \%)$ e casados (56\%). Foram atendidos pacientes de todos os níveis educacionais, sendo que $59 \%$ haviam concluído apenas o ensino médio e $34 \%$ o nível superior. A renda da maioria dos pacientes era de 10 salários mínimos (60\%), sendo $23 \%$ com renda de até dois salários mínimos. Dezesseis por cento dos pacientes recebiam mais de 40 salários mínimos.

Dos participantes, a maioria (87\%) aceitou bem apresença do residente, que foi predominantemente do sexo feminino (66\%). Um paciente (1\%) não gostou da presença do residente durante a consulta e exame proctológico, enquanto onze por cento consideraram tal presença indiferente. Um paciente não respondeu (Figura 1).

Dentre aqueles que aceitaram a presença do residente, a maior parte (48\%) assinalou como motivo principal para tal aceitação o fato de estarem contribuindo para a formação médica especializada. Outros fatores assinalados para aceitação dos residentes foram a sensação de maior segurança pela presença de dois médicos (44\%), o entendimento que os médicos mais jovens, em período de formação, costumam ser mais interessados (28\%) assim como a sensação de maior conforto pelo fato de o residente ser do mesmo sexo (27\%) (Tabela 2).

Embora apenas um paciente não tenha aprovado a presença do residente durante a consulta e exa- me proctológico, outros dois pacientes indiferentes com relação à presença do residente também assinalaram motivos para sua recusa. Tais motivos foram o fato de sentire-se envergonhados com a presença do residente $(67 \%)$ e por perderem a privacidade $(33 \%)$. Nenhum destes pacientes manifestou, espontaneamente, o desejo que o residente se retirasse antes do início da consulta.

Não houve diferença estatisticamente significativa na correlaçao entre a aceitação do residente e o sexo, a raça, a idade, a escolaridade e a renda familiar dos pacientes. Com relação ao estado civil, um maior número de pacientes casados aceitou a presença do residente durante a consulta e exame proctológico $(p=0,035)$.

A influência do sexo do residente também foi estudada e não houve correlação estatisticamente significativa entre esta variável e aceitação ou recusa de sua presença por parte dos pacientes. A maioria dos pacientes não alteraria suas atitudes com relação à presença do residente,caso ele fosse do sexo oposto (Tabela 3).

No que diz respeito a outras questões relacionadas à visão do paciente quanto à presença do residente durante o exame proctológico, observou-se que apesar de $11 \%$ dos pacientes serem indiferentes a este aspecto, todos eles prefeririam ser examinados sem a presença do residente. Por outro lado, a maioria destes pacientes voltaria sabendo da presença do residente ou permitiria ser atendido pelos residentes. Entretanto, nenhum destes pacientes permitiria ser examinado pelo residente (tabela 3 ).

\section{DISCUSSÃO}

Este estudo demonstrou que, na maioria das vezes, o residente foi bem aceito em um ambulatório de coloproctologia de instituição privada. Os fatores

Tabela 2 - Razões para a aceitação da presença do residente durante a consulta e exame proctológico $(n=87)$.

\begin{tabular}{lll}
\hline Razões para aceitação da presença do residente & n* & \% \\
\hline Contribuição para a formação médica & 42 & 48 \\
Maior segurança pela presença de dois médicos & 38 & 44 \\
Médicos mais jovens são mais interessados & 24 & 28 \\
Maior conforto por residente ser do mesmo sexo & 24 & 28 \\
\hline
\end{tabular}

* A maioria dos pacientes assinalou mais de uma razão. 
Tabela 3 - Questões formuladas aos pacientes com relaçãoo à presença do residente durante exame proctológico realizado em ambulatório.

\begin{tabular}{lccc}
\hline Questões relacionadas à presença do residente & Sim & Não & Não respondeu \\
\hline Você preferiria ser examinada sem a presença do residente? & 13 & 87 & 0 \\
Voltaria sabendo da presença do residente? & 94 & 4 & 2 \\
Deixaria ser examinada pelo residente? & 87 & 10 & 3 \\
Se negaria a ser atendido com a preseça do residente? & 3 & 93 & 4 \\
Mudaria as respostas se o residente fosse de outro sexo? & 12 & 86 & 2 \\
\hline
\end{tabular}

que mais influenciaram a aceitação do residente pelos pacientes foram a possibilidade de contribuir para a formação médica e a sensação de maior segurança ao serem examinados por dois médicos. Estudo semelhante, avaliando os fatores que influenciavam a opinião de pacientes de consultórios obstétricos e ginecológicos em relaçaõ à presença de estudantes de medicina, evidenciou que o fator que mais influenciou na decisão do paciente para a aceitação do estudante foi o desejo em contribuir para o treinamento de futuros médicos ${ }^{1}$.

Em outro estudo que pesquisou as atitudes de pacientes de clínica dermatológia em relação a presença de estudante de medicina, observou-se que muitos dos pacientes comentaram que seria um bom treinamento para os estudantes, por ser uma grande oportunidade para aprendizado prático ${ }^{2}$.

A sensação de vergonha por exposição íntima foi a maior causa de recusa, do residente no presente estudo. Em trabalho semelhante ao nosso estudo, também realizado em ambulatório de coloproctologia, porém com a presença de estudantes de medicina ao invés de residentes, obtiveram como maiores causas de recusa da presença dos estudantes, a perda de privacidade e o aumento do tempo da consulta ${ }^{3}$. Esta última razão não foi assinalada por nenhum paciente no presente estudo.

Com relação às atitudes de mulheres em relação ao sexo de estudantes de medicina em clínica ginecológica, observou-se que havia uma preferência por estudante do sexo feminino tanto na observação do exame ginecológico quanto na realização do mesmo ${ }^{4}$. Estudo semelhante avaliou a preferência de homens em relação ao sexo do médico no exame genital e retal e evidenciou que $51 \%$ dos pacientes tinham preferência pelo sexo masculino quando se tratava do exame genital, contra apenas $38 \%$ quando se tratava do exame retal. Apesar de o restante considerar indiferente o sexo do examinador, nunhum paciente expressou claramente preferência por profissional do sexo femini- no ${ }^{5}$. No presente estudo não houve correlação estatisticamente significativa entre o sexo do residente e a aceitação ou recusa da presença do mesmo por parte dos pacientes, sendo que a maioria não alteraria suas atitudes com relação à presença do residente, caso ele fosse do sexo oposto.

No que diz respeito ao tipo e magnitude da doença apresentada pelo paciente foi demonstrado que, em uma clinica de medicina geral, todos os pacientes aceitariam bem a presença do estudante de medicina, caso seu problema fosse uma amigdalite, enquanto $70 \%$ concordariam se o problema fosse emocional. A taxa de aceitação cairia ainda mais (50\%), se o problema fosse sexual ou se fosse necessário um exame íntimo ${ }^{6}$.

O Consentimento informado do paciente para a participação do residente em sua consulta, não foi obtido no nosso estudo, o que talvez pudesse ter influenciado sua opinião. O Colégio Real de Obstetras e Ginecologistas da Inglaterra defende que o consentimento explícito deve ser obtido das pacientes para que estudantes de medicina participem e/ou realizem exame intimo ${ }^{7}$.

A identificação dos fatores relacionados à aceitação da presença do residente no ambulatório de coloproctologia pode contribuir para que a postura médica, não só residente, mas principalmente do preceptor, enfatize atitudes positivas e minimize atitudes negativas, de forma a facilitar a participação do residente na consulta e exame proctológico.

Atenuar as razões negativas pelas quais os pacientes não aceitam a participação do residente é tarefa árdua, devido às especificidades de nossa especialidade. Portanto, assegurar privacidade e conforto ao paciente durante seu exame torna-se fundamental, particularmente na presença do residente, que deve ser sempre apresentado como tal pelo preceptor. Sua permanência durante a consulta e o exame proctológico deve ser autorizada pelo paciente ou seu responsável. 


\begin{abstract}
Residents exposure to patientes and their participation in the examination is essential for the learning of colorectal surgery specialty. Patients may be reluctant to have residents present in outpatient colorectal clinics. This study evalueted patients' attitudes towards resident' $s$ involvement in an outpatient colorectal clinic of a private institution. Methods: One hundred consecutive patients answered voluntarily and anonymously a questionnaire after the physical examination with the involmement of residents. The examination consisted of anal inspection, palpation, anoscopy, rigid proctosigmoidoscopy and abdominal examination. Age, gender, race, marital, educational and social status were evaluated, as well as the patients' attitudes towards resident's involvement in the outpatient setting. The reasons for acceptance or refusal of resident' involvement werw also evaluated. For statistical analysis the [chi] $]^{2}$ os Fisher test and $t$ Student test were used. Results: The acceptance rate of residents during the rectal examination was 87 per cent and the refusal rate was 1 per cent. Eleven per cent reported tha resident's involvement was indifferent for them and 1 patient did not answer this question. There was no significant difference among the studied variables regarding resident's involvement, except for the marital status, since married patients accepted the resident better than single patients. The main reason for acceptance was cooperatin for medical training, while for refusal was loss of privacy. Conclusion: Patients accept resident's involvement during rectal examination in outpatient setting. Knowledge of the reasons for their acceptance and refusal by patients may emphasize positive attiyudes in order to improve this acceptance and minimize the refusal.
\end{abstract}

Key words: resident, rectal exam, medical training.

\section{REFERÊNCIAS}

1. Ching SL, Gates EA, Robertson PA - Factors influencing obstetric and gynecologic patients' decisions toward medical student involvement in the outpatient setting. Am J Obstet Gynecol 2000; 182:1429-1432

2. Townsend B, Marks JG Mauger DT, Miller JJ - Patients' attitudes toward medical student participation in a dermatology clinic. J.Am Acad Dermatol 2003; 49:709-711

3. Shah-Khan M - Patient Attitudes toward Medical Students in an Outpatient Colorectal Surgical Clinic. Dis Colon Rectum 2004;47:642-643

4. O'Flynn N, Rymer J - Women's attitudes to the sex of medical students in a gynaecologic clinic: cross sectional survey. BMJ 2002;325:683-684
5. Heaton CJ, Marquez JT - Patient preferences for physician gender in the male genital/rectal. Fam Pract Res J 1990; 10:105-15

6. O'Flynn N, Spencer J, Jones R - Consent and confidentiality in teaching in general practice: survey of patient' views on presences of students. BMJ 1997;315:1142.

7. Royal College of Obstetricians and Gynaecologists - Intimate Examinatios. Report of a Working Party. London: RCOG Press, 1997.

Endereço para correspondência:

ISABELA PESSOA ELIAS

Rua Uberaba, 418/504 - Barro Preto

Belo Horizonte - M.G.

30180-080

E-mail: Isabelaelias@ Hotmail.com 